hemorrhage. Throughout the book, Drs. Caplan and Kase have emphasized the historical evolution of our current concepts and theories on intracerebral hemorrhages. The lead authors, both experts in this field, have written all the chapters on the mechanisms, clinical features, location, and treatment of intracerebral hemorrhage. Additional chapters have been contributed by authorities in neuropathology, neuroimaging, epidemiology, and neurosurgery.

The book is divided into four major sections. The first section deals with the general features of intracerebral hemorrhage and opens with an entertaining and instructive chapter providing a historical overview. Subsequent chapters on epidemiology, general signs and symptoms, pathology, and modern neuroimaging provide the necessary background for those readers lacking experience in this field. The discussion on the signs and symptoms provides clinical pearls on the presentation of intracerebral hemorrhage often neglected in this era of neuroimaging.

The second section is devoted to mechanisms of intracerebral hemorrhage. The chapters on hypertensive hemorrhages, amyloid related hemorrhages, and aneurysms and vascular malformations provide a wealth of clinically relevant material. A chapter on vasculitis and other angiopathies is particularly valuable as it assembles available knowledge on several unusual causes of intracerebral bleeding that is difficult to find in a single source. Other topics include bleeding disorders, drugs, trauma, and tumors.

Part three deals with the clinical features of intracerebral bleeding at different sites. Anatomical drawings, pathological photos, and examples of CT or MRI findings complement the text. Brief case descriptions are frequently used to add depth to the discussions. Most chapters conclude with an analysis of outcome variables and current treatment.

Part four is an overview of the prognosis of $\mathrm{ICH}$ and the medical and surgical therapy. The surgical discussion focuses predominantly on the issue of hematoma evacuation and ventriculostomy.

This is an excellent book which admirably fulfills its objectives. It is well written and I found it easy and enjoyable to read. It is a book written by clinical experts for use by clinicians. Each chapter is well referenced and the index is adequate and useful. The discussion on the treatment of AVMs and vascular malformations could have been expanded. Little information has been provided on the role of the "gamma knife", radiosurgery, and interventional neuroradialogical therapy. Except for these shortcomings, the book is quite thorough without being exhaustive or tedious. I would strongly recommend it to neurologists, neurosurgeons, and residents with an interest in vascular diseases of the nervous system.

Philip A. Teal Vancouver, British Columbia.

ANTIEPILEPTIC DRUGS, FOURTH EDITION. 1995. Edited by René H. Levy, Richard H. Mattson and Brian S. Meldrum. Published by Raven Press. 1148 pages. $\$ C 233.00$

There is no more rapidly expanding field in neurological therapeutics than that of antiepileptic drugs. We have witnessed the release of three new drugs in the last two years: vigabatrin, gabapentin and lamotrigine, with others such as topiramate, tiagabine and oxcarbazepine on the horizon. It is safe to predict that the pharmacological treatment of epilepsy will be quite different from our present approach ten years hence. The fourth edition of Antiepileptic Drugs has expanded considerably over its predecessor, now to 98 chapters and 1120 pages, reflecting the growing knowledge in this area.
This book in its earlier editions has become the reference "bible" for information on antiepileptic drugs, their development, pharmacology and clinical use. The present edition maintains the very high standard of content and production and will serve as a suitable reference source for neurologists, epileptologists, pharmacologists and pharmacists on all aspects of these agents. The first section, "General Principles", includes excellent chapters on pharmacolinetics, experimental aspects, clinical trial design, compliance, principles of drug selection, use of serum levels, teratogenicity, discontinuation of drugs and use of antiepileptic drugs in the elderly. This is followed by sections on the individual drugs including those which are no longer used such as bromides, trimethadione or progabide. Newer drugs and drugs under development are given ample coverage with recent data. The chapters in each section follow an orderly progression: mechanisms of action; chemistry and biotransformation; absorption, distribution and excretion; interactions; clinical use and tosicity. This well-organized format in addition to an extensive index facilitates location of specific information on a particular drug.

This volume is truly encyclopedic in scope but the editors have done a superb job in making the material from multiple authors cohesive and readable. This book should find its way onto the shelf of every epileptologist and epilepsy researcher. It should also be readily accessible to neurologists psychiatrists, neurosurgeons working in epilepsy, pharmacologists and hospital pharmacists. Neurology trainees could benefit greatly from reading several of the chapters in the "Principles" section. I congratulate the editors on providing such a usable and up-to-date reference in this burgeoning field.

A. Guberman

Ottawa, Ontario

MULTIPLE SCLEROSIS FACT BOOK. SECOND EDITION. 1995. By Richard Lechtenberg. Edited by Robert W. Reinhardt, Bernice M. Wissler and Glenn L. Fechner. Published by F.A. Davis Company. 235 pages. $\$ C 26.00$

The Multiple Sclerosis Fact Book is precise, accurate and current. Dr. Richard Lechtenberg, the author, has addressed Multiple Sclerosis and its effects in easily understood terms. Clearly defined illustrations support these facts. People who have Multiple Sclerosis; those who care; the interested general public and medical personnel will benefit from this wealth of information.

Lechtenberg presents an extensive overview of what Multiple Sclerosis is; who is commonly affected and how diagnosis is determined. The author also discusses the social, psychological and sexual problems which may be encountered. In addition, explanations of lifestyle adjustments and methods of rehabilitation are offered.

Sections on "Research Efforts", "Recommended Reading" and a "Glossary of Terms" conclude this detailed, informative work.

As a comprehensive resource on the subject of Multiple Sclerosis, this is a must read.

Lynn Flynn Calgary, Alberta

ATLAS OF BRAIN FUNCTION. 1995. By William W. Orrison, Jr. Published by Thieme Medical Publishers, Inc. 112 pages. $\$ C 77.00$

This brief atlas of the brain is divided into two sections: the first is a glossary of anatomic structures with a description of 
their location and function/functional relationships, which covers 30 pages. The second section contains sagittal, axial and coronal CT and MR images, and a small section of 3D images with corresponding line drawings.

The Glossary section is succinct and is the only strength of this book. The descriptions in this section would be appropriate for an audience of undergraduate level. Unfortunately, the second section of the book contains CT and MR images of disappointingly low quality. Many of the structures labelled on the corresponding line diagrams can hardly be visualized on the cross-sectional images. In the Preface, the authors state that the atlas is intended as a reference book, but it is of insufficient detail to serve that purpose, and would, at best, be of limited use as an introductory learning tool for undergraduate medial trainees.

William $\mathrm{Hu}$

Toronto, Ontario

\section{DIFFUSION AND PERFUSION MAGNETIC RESONANCE IMAGING: APPLICATIONS TO FUNCTIONAL MRI. FIRST EDITION. 1995. Edited by Denis Le Bihan. Published by Raven Press, Ltd. 374 pages. \$C207.00}

There are few books currently available which deal exclusively with diffusion and perfusion imaging by Magnetic Resonance Imaging (MRI). Most of the information available for physicists and imagers is to be found either as sections of current texts on MRI, or journal articles. This book brings together accumulated experience with diffusion and perfusion MRI.

Edited by one of the experts in the field, and with contributions from most, if not all of the leaders researching this subject, the book reviews current knowledge of diffusion and perfusion imaging using Magnetic Resonance Imaging, including blood oxygen level dependent -BOLD- imaging or functional MRI.

The first section of the book deals with the physics of diffusion imaging, the measurement of molecular movement and displacement in tissue, as done by MRI. Chapters in this section deal with acquisition of diffusion data, using various pulse sequences (spin echo, stimulated echo, echo planar and steady state free precession), and the theory behind such sequences. There is an excellent chapter on hardware requirements for the different types of sequences, which is well written and illustrated. Also in this section is a highly technical chapter discussing the derivation of the $b$ factor, from which the Apparent Diffusion Coefficient (ADC) is derived, and a very complex appendix dealing with computer codes used to derive the $b$ matrix. The section contains chapters dealing with motion artifacts that may occur in diffusion MRI, with a good discussion of ways to reduce or correct the artifacts, as well as effects of magnetic susceptibility variations which may degrade image or distort diffusion data. Last in this section are chapters on clinical applications, with appropriate images, and a short section on temperature imaging by MR.

The second section, on perfusion imaging, deals with physics of the delivery of blood to areas within the brain at a capillary level. It discusses various models of tracer kinetics, as well as exogenous contrast agents, and magnetic labelling. The clinical chapters in this section deal mostly with stroke and brain tumor, but also mention Alzheimer's disease, and compare the MRI findings with traditional methods of perfusion imaging such as PET. Most of the applications described for spin labelling, or flow-compensated/flow dephased imaging, however, deal with animals with various models of disease. Also contained in this section are chapters on brain function mapping, which are very well written, and are recommended reading for newcomers to functional MRI.

The last section of the book deals with the current hot topic in MRI - functional MRI using BOLD imaging. After a discussion of the actual BOLD effect, clinical applications are discussed. While there are no color images directly in this section, there is a central collection of color plates in the book which are of excellent quality showing representative clinical images, and black and white images are in this section.

In general, the book is well written, illustrated and referenced. Only a few sections may be out of place - one chapter on diffusion imaging of the kidney is included in perfusion imaging, and the chapter on brain function mapping in the section on perfusion imaging might have been better included in the last section on BOLD imaging.

As an imager, there were sections that I found highly technical, difficult to read and understand, and of little relevance to clinical practice. However, the physicists in our research group were excited by the physical aspects of diffusion and perfusion imaging covered in the book. Sections I found particularly useful were those on functional MRI, and clinical applications of brain diffusion imaging.

This book will thus form a seminal reference manual for researchers involved in diffusion and perfusion imaging with MRI.

Donald H. Lee

London, Ontario

CEREBRAL SPECT IMAGING, 2ND EDITION. 1994. Edited by R.L. Van Heertum and R.S. Tikofsky. Published by Raven Press. 233 pages. $\$ \mathrm{C} 182.00$

The current volume has been significantly expanded since the first edition which was published in 1989. The emphasis is on SPECT brain perfusion imaging which should be available clinically in most centres. PET studies and receptor imaging which often form the major part of reviews on the topic are mentioned only by way of comparison. Introductory chapters discuss instrumentation, radiopharmaceuticals, and normal gross and functional anatomy. The coverage is concise but superficial, and specialists in Nuclear Medicine and referring clinicians in the Neurosciences who are the target audience identified in the preface, will wish to be selective in their reading based on their areas of expertise. References are not comprehensive but do include major reviews of the various topics.

The main strength of the book is as a clinical atlas which includes over 100 well documented cases, illustrating findings and uses in evaluation of cerebrovascular disease, dementia, seizure disorders, trauma, and psychiatric conditions. Each chapter begins with a summary of key features and is followed by a series of clinical cases. There is good correlation with $\mathrm{CT}$ and MRI, and followup studies where appropriate. Particular teaching points are highlighted for most cases. 